\title{
Fatigue Performance of the Novel Titanium Alloy TIMETAL ${ }^{\circledR} 407$
}

\author{
W. Davey ${ }^{1}$, M.R. Bache ${ }^{1, *}$, H.M. Davies ${ }^{1}$, M. Thomas ${ }^{2}$ and I. Bermant-Parr ${ }^{2}$ \\ ${ }^{1}$ Institute of Structural Materials, College of Engineering, Bay Campus, Swansea University, Swansea, SA1 8EN, UK \\ ${ }^{2}$ Timet UK Ltd, PO Box 704, Witton, Birmingham, B6 7UR, UK \\ *Corresponding author: m.r.bache@swansea.ac.uk
}

\begin{abstract}
$\underline{\text { Abstract }}$
TIMETAL ${ }^{\circledR} 407$ (Ti-407) is a novel titanium alloy formulated as a medium strength, highly ductile alloy offering a range of manufacturing cost reduction opportunities. It can be used as a direct replacement for Ti-6Al-4V or Ti-3Al-2.5V alloys, particularly in applications where energy absorption during fracture or HCF endurance are the key design criteria. The effect of thermo-mechanical processing on microstructure has been characterised and the room temperature high cycle, low cycle and dwell cycle fatigue properties of Ti-407 containing 30-40\% primary alpha volume fraction are presented and discussed. These results are compared with data generated from Ti-6Al-4V processed to provide a similar but non-standard microstructure and demonstrate that Ti-407 shows superior HCF endurance strength, despite having a significantly lower tensile and yield strength.
\end{abstract}

\section{$\underline{\text { Introduction }}$}

Titanium alloys have been employed extensively within the aero-engine sector since the $1950 \mathrm{~s}$. The high strength to weight ratio at low to intermediate service temperatures, good corrosion resistance and low density relative to that of competing metals, contribute to the production of lightweight and durable components with applications ranging from the large-scale fan blades and supporting discs at the front of the engine, through to relatively small vanes and blades in the high pressure compressor section. The well-established Ti-6-4 (Ti-6Al-4V) is still used for a large number of engine components, in part due to the range of microstructural forms produced via thermo-mechanical processing that can be tailored to specific applications. Ti-6-4 accounts for $50 \%$ of the total titanium production ${ }^{[1]}$. This alloy is widely acknowledged as presenting a balance of mechanical properties including good fatigue performance with intermediate fracture toughness and moderately high tensile strength. Over the years much work has been performed to optimise the manufacturing and production of Ti-6-4 components. However, economic competition is encouraging more cost-effective production, leading to the development of bespoke alloys better suited for specific components in terms of both mechanical performance and ease of manufacture.

Titanium alloys with lower aluminium content than Ti-6-4 are notably more malleable. For example, it has been shown previously that an alloy of composition Ti-1Al-4V provided a UTS of approximately $650 \mathrm{MPa}$ and an elongation to failure of $25 \%{ }^{[2]}$. This knowledge was taken as the starting point for the development of the new commercial alloy Ti-407. During the alloy development stages it was important to demonstrate a viable and economical solution which considered factors such as the availability of raw materials, the preferred melting method, the effect of industrial conversion routes and the properties of heavily forged sections. Thermodynamic tools were used to down-select a range of potential alloy compositions and laboratory scale thermo-mechanical processing was used to produce production representative sub-scale specimens for detailed mechanical testing. Multiple linear regression calculations and neural network models were used to help optimise the most promising chemistries. Table I defines the composition and physical properties of the eventual Ti-407 chemistry relative to alternative Ti-6-4 and Ti-3-2.5 commercial products.

It is proposed that Ti-407 could replace Ti-6-4 in applications where the key design criteria are energy absorption during fracture and HCF endurance strength. The most relevant applications within the gas turbine are, therefore, those structural applications where impact resistance is important. Previously published mechanical assessments showed that Ti-407 absorbed nearly twice the impact energy of Ti-6-4 and exhibited 2.5 times the lateral expansion under representative loading during Charpy tests ${ }^{[3]}$. Further to this, Ti-407 displayed more than double the tool life than that of Ti-6-4, based on V15 tests (a standardized machining test that allows ranking between alloys, V15 referring to the speed of a cutting tool that induces complete wear in a period of 15 minutes). The reduction in tool wear supports lower forces required for faster, more efficient machining. Compared to Ti-6-4, the relatively low elevated temperature flow stress, greater malleability and wide process window should allow Ti-407 to be processed with fewer reheats, while exhibiting a consistently good surface finish with reduced surface cracking. Optimised Ti-407 manufacturing processes should allow parts to be formed closer to net shape giving higher yields and actually requiring less machining to finished size. Whilst Ti-407 is slightly denser than Ti-6-4, the superior impact energy capability should offer potential component weight savings.

Table I. Nominal composition and physical properties of Ti-407, Ti-6-4 and Ti-3-2.5.

(C) The Authors, published by EDP Sciences. This is an open access article distributed under the terms of the Creative Commons Attribution License 4.0 (http://creativecommons.org/licenses/by/4.0/). 


\begin{tabular}{|l|c|c|c|}
\hline Attribute & Ti-407 & Ti-6-4 & Ti-3-2.5 \\
\hline $\mathrm{Al}, \mathrm{wt} \%$ & 0.85 & 6 & 3 \\
\hline $\mathrm{V}, \mathrm{wt} \%$ & 3.1 & 4 & 2.5 \\
\hline $\mathrm{O}, \mathrm{wt} \%$ & - & 0.15 & 0.1 \\
\hline $\mathrm{Si}, \mathrm{wt} \%$ & 0.31 & - & - \\
\hline $\mathrm{Fe}, \mathrm{wt} \%$ & 0.22 & 0.15 & - \\
\hline $\mathrm{\rho}, \mathrm{g} \mathrm{cm}^{-3}$ & 4.53 & 4.42 & 4.48 \\
\hline $\mathrm{T} \beta,{ }^{\circ} \mathrm{C}$ & 877 & 996 & 935 \\
\hline
\end{tabular}

\section{Experimental Methods}

\section{Ti-407 Production and Sampling}

Pre-production Ti-407 material (Ti-0.85Al-3.9V-0.15O-0.25Si-0.25Fe) was produced by TIMET via electron beam single melting (EBSM) in a cold hearth furnace to create an ingot and then processed using a combination of beta forging, plus alpha-beta forging or rolling ${ }^{[3]}$. The present study then utilised "pancake" forgings produced from this material. Billet sections were cut into four, $90^{\circ}$ degree segments. These were then flat die forged into the pancake form, and mechanical test specimen blanks were extracted from the central regions.

Development of a suitable microstructure is an important consideration to control the mechanical performance of titanium alloys. Increasing primary alpha $\left(\mathrm{a}_{\mathrm{p}}\right)$ content has been shown to increase tensile plasticity ${ }^{[4]}$, refine the microstructure and limit the formation of areas of common orientation ${ }^{[5]}$. Whilst $a_{p}$ within a lamellar matrix does offer these advantages, the percentage volume must be carefully restricted since beyond a critical volume, ductility ceases to improve and alloy element partitioning takes place. This degrades the basic strength of the lamellar constituent of the microstructure, therefore resulting in a decrease in fatigue life. Following heat treatment trials to determine the most suitable temperature, all mechanical Ti- 407 specimen blanks were solution treated at $840^{\circ} \mathrm{C}$ for two hours and air cooled, followed by an eight-hour aging treatment at $500{ }^{\circ} \mathrm{C}$ and air cool. The resulting 30\% volume fraction of $a_{p}$ grains demonstrated an average $a_{p}$ grain diameter of 5 to 10 microns (Figure 1a).

The Ti-6-4 test pieces were processed via two distinct heat treatment routes, both non-standard and designed to produce a similar microstructure to that of the Ti-407 specimens in order to allow for an academic comparison. In both cases the specimen blanks were solution treated at $960^{\circ} \mathrm{C}$ for two hours and air cooled. One set was then aged at $500^{\circ} \mathrm{C}$ for 8 hours and air cooled as with the Ti-407 blanks, Figure 1b. The second set was annealed at $700^{\circ} \mathrm{C}$ for two hours and air cooled, Figure 1c. In the aged Ti-6-4 microstructure, the average diameter of the primary alpha grains varied between 15-30 microns. For the Ti-6-4 annealed test pieces, the typical diameter of the primary alpha grains was moderately smaller at 10-20 microns.
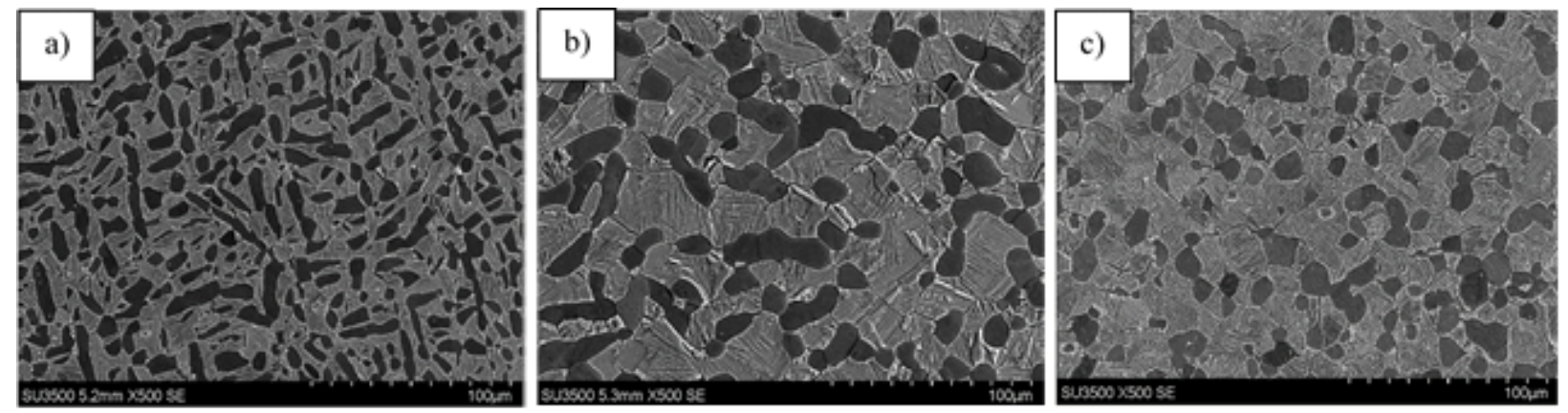

Fig. 1. Typical microstructures, a) Ti-407, b) Ti-6-4 aged and c) Ti-6-4 annealed.

The blanks were then machined using CNC turning operations into plain cylindrical specimens suitable for tensile and fatigue experiments. A non-contacting shadowgraph was employed to measure the co-axiality of the test pieces and the critical dimensions of the parallel gauge section.

\section{HCF, LCF and Dwell Testing}

All mechanical test data refer to room temperature assessments. High cycle fatigue (HCF) tests were conducted on an Amsler vibrophore resonance machine at a load ratio of $\mathrm{R}=0$, using a sinusoidal waveform with a frequency of $100 \mathrm{~Hz}$. Low cycle fatigue (LCF) tests, again under $\mathrm{R}=0$ were performed on various servo-hydraulic test rigs employing a 15 cycle per minute trapezoidal waveform, comprising one second linear rise and fall ramps with a one second hold at both peak and minimum load. Dwell tests 
were also carried out on servo hydraulic test rigs, with a 120 second hold at peak stress. In all fatigue tests, complete rupture of the specimen was induced (i.e. no "run-outs" were generated).

\section{$\underline{\text { Results }}$}

\section{Tensile Testing}

Tensile properties of the Ti-407 and Ti-6-4(aged) variants were measured during the current project employing an applied strain rate of $0.002 / \mathrm{min}$ to define yield, increasing to a rate of $0.01 / \mathrm{min}$ to induce fracture. The results are summarised in Table II and compared to data for the alloy Ti-3-2.5 ${ }^{[6]}$.

Table II. Tensile data for Ti-407, Ti-6-4(aged) and Ti-3.2.5.

\begin{tabular}{|l|c|c|c|}
\hline Attribute & Ti-407 & $\begin{array}{c}\text { Ti-6-4 } \\
\text { (aged) }\end{array}$ & Ti-3-2.5 \\
\hline Elastic modulus (GPa) & 114 & 116 & 100 \\
\hline Tensile strength (MPa) & 764 & 1037 & 620 \\
\hline Yield strength (MPa) & 648 & 869 & 500 \\
\hline Ductility (\%) & 19 & 12 & 15 \\
\hline
\end{tabular}

\section{Fatigue Data and Fractography}

The comparative HCF performance of Ti-407 and both the aged and annealed variants of the non-standard Ti-6-4 are illustrated in Figure 2. Least squares best fit regression lines are superimposed for the individual data sets. The data indicate a superior endurance strength (i.e. defined at $\mathrm{N}>10^{7}$ cycles) for Ti-407 of approximately 100MPa when compared to either variant of Ti-6-4.

Concentrating on Ti-407 alone, HCF, LCF and dwell data are plotted in Figure 3, with least squares best fit regression lines superimposed for each individual dataset. Notably, the range of applied maximum cyclic stress assessed under either regime was identical (with individual tests performed across the range 550 and 650MPa). This helps to emphasise the significantly stronger response under HCF conditions relative to LCF and in turn dwell performance.

Particular attention was afforded to the individual Ti-407 HCF fracture surfaces. These displayed a mix of surface and sub-surface initiation sites (indicated by hollow and solid data points in Figure 3 respectively). The sub-surface initiated failures were easily identified by eye, Figure 4a. A near circular, halo was usually evident, formed by the sub-surface crack whilst it propagated in a radial fashion under a vacuum environment. The fracture surface surrounding the halo, formed subsequent to the crack breaking through at the surface and now open to oxidation, displayed a dull appearance. Under high magnification scanning electron microscope (SEM) inspection, Figure 4b, quasi-cleavage facets were invariably revealed at the centre of the halo marking the precise site of fatigue crack initiation. The facets were evident as a cluster of elongated features, relatively dark under secondary electron view, representing the earliest stages of fracture through neighbouring $a_{p}$ grains. These were surrounded by regions of a more ductile appearance, representing failure of the adjacent transformed $a / \beta$ microstructure.

\section{Discussion}

From the perspective of ranking fatigue performance, Ti-407 demonstrated significantly stronger fatigue endurance strength compared to both aged and annealed forms of the non-standard Ti-6-4 under HCF loading, Figure 2. Under relatively high applied stress conditions, the annealed variant of Ti-6-4 demonstrates superior performance to that of the aged variant, attributed to the finer microstructure evolved during the annealing heat treatment process, Figure 1. At lower stresses the fatigue response of the two Ti-6-4 forms was very similar. The Ti-407 endurance strength defined at $10^{7}$ cycles is approximately 100MPa greater than both forms of Ti-6-4. Therefore, together with superior ductility, machinability and manufacturing properties, Ti-407 should be viewed as a promising alternative alloy for aero-engine containment structures or any engineering application where vibrational loading is expected to dominate. 


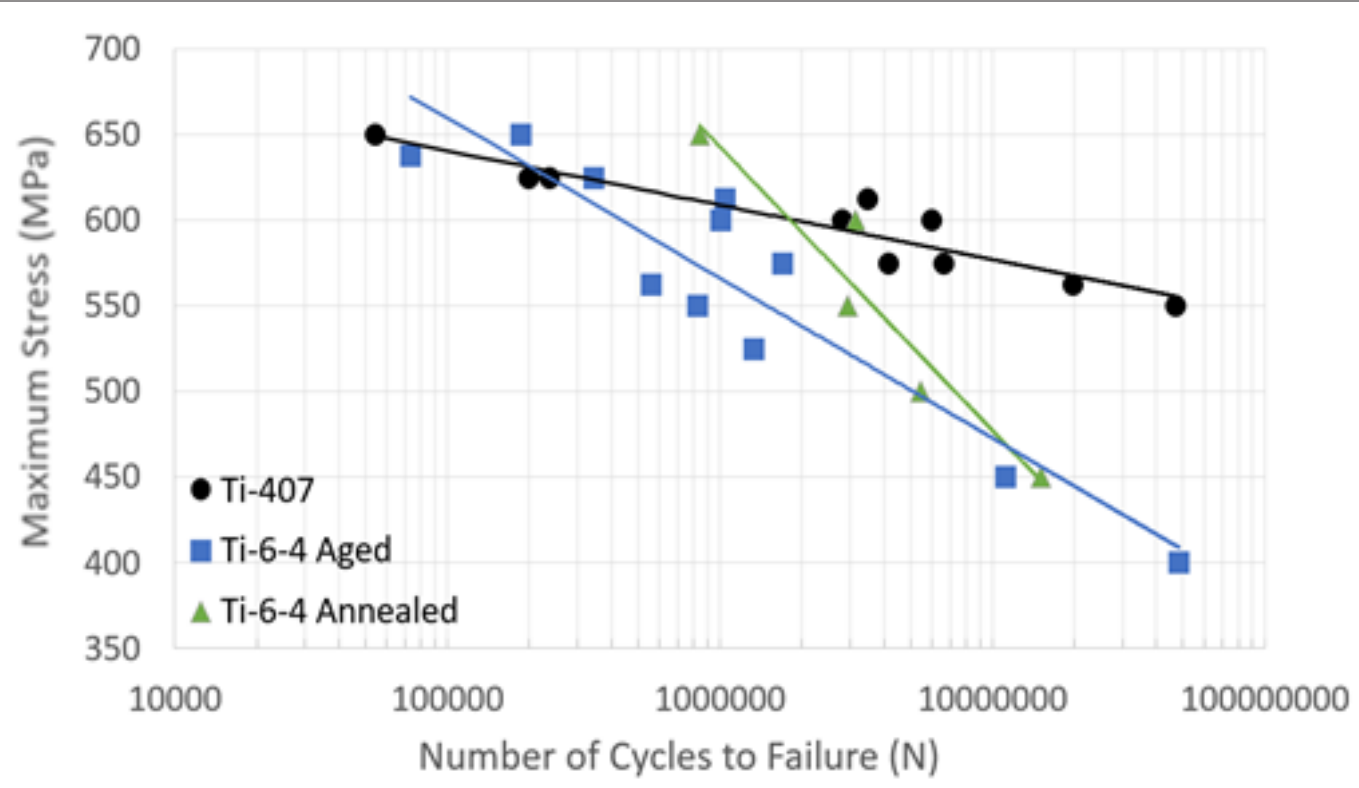

Fig. 2. HCF data for Ti-407 vs Ti-6-4 (aged and annealed), $R=0$, room temperature

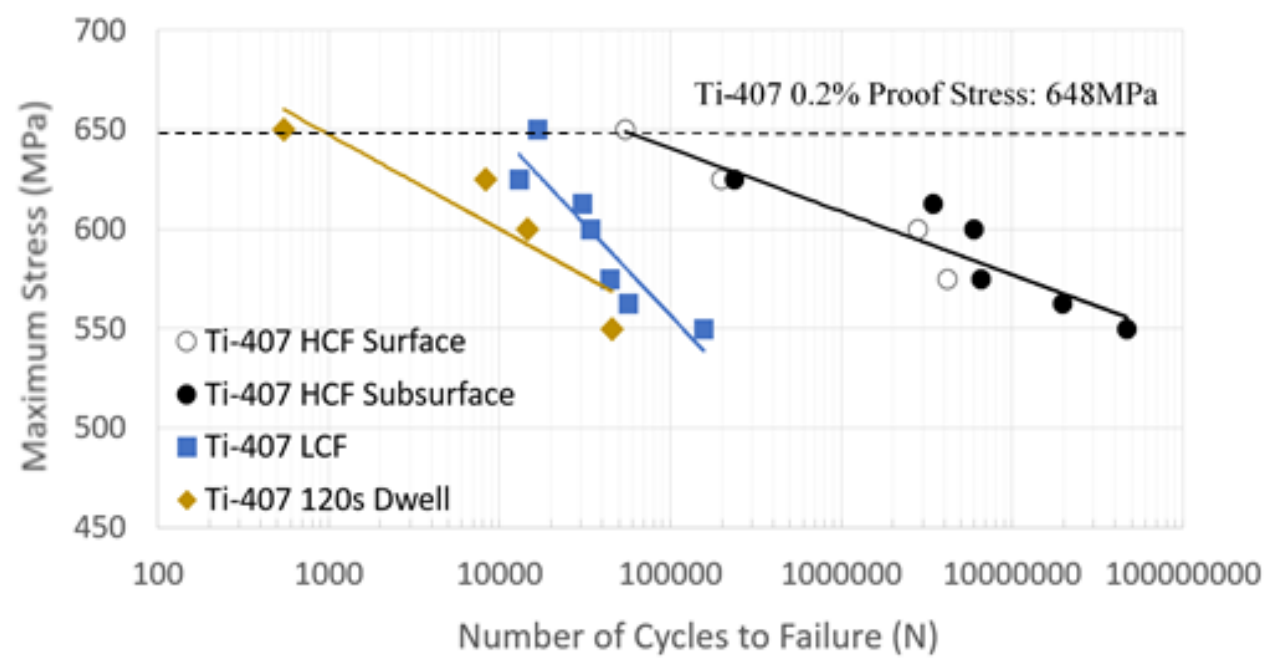

Fig 3. HCF, LCF and dwell fatigue data for Ti-407, $R=0$, room temperature.
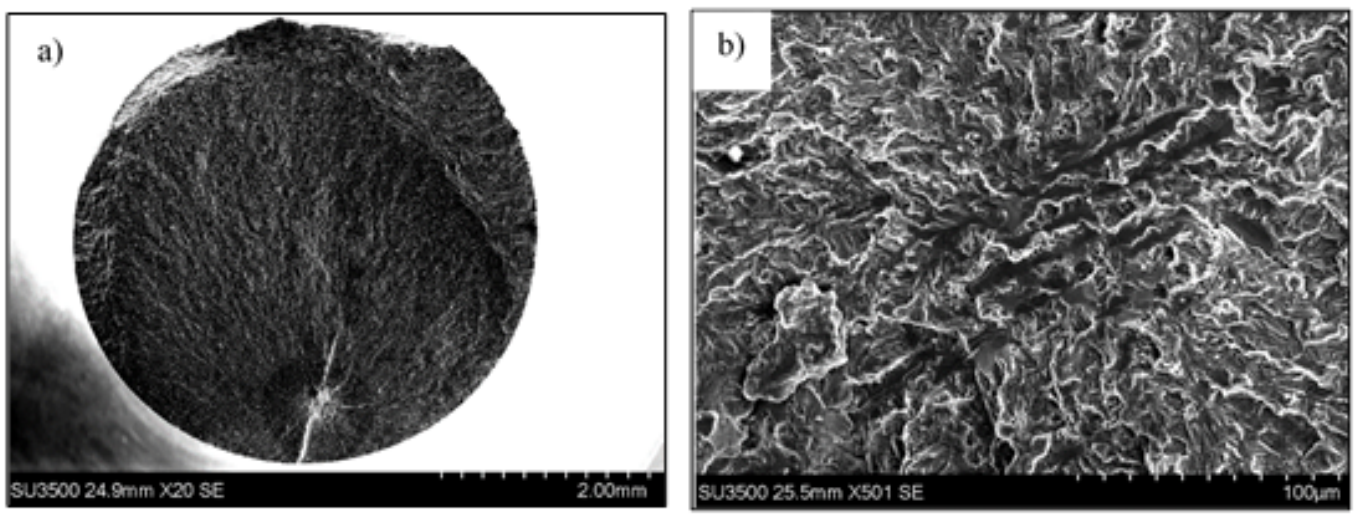

Fig. 4. a) Macroscopic view of a typical Ti-407 HCF fracture surface; b) cluster of elongated facets at initiation site.

A single solution heat treatment was adopted for the Ti-407 specimens assessed under HCF, LCF and dwell conditions during the present study, producing an $\mathrm{a}_{\mathrm{p}}$ volume fraction of 30\%. Regardless, the relatively high HCF strength of Ti-407 is an encouraging outcome for what is recognised as a comparatively low strength alloy under static loading. In that context, it is interesting to compare the performance of Ti-407, to literature data describing Ti-3Al-2.5V (another relatively weak alloy under monotonic tension) offering UTS of $620 \mathrm{MPa}$ and HCF endurance strength of $280 \mathrm{MPa}$ measured at $10^{7}$ cycles $^{[6]}$. Thereby considering the ratio between endurance and ultimate strengths, Ti-3Al-2.5V offers a ratio at approximately 0.45 , whereas the equivalent performance of Ti-407 was found to approach 0.75 . Many different strength variants of Ti-6-4 can be found amongst the open 
literature, however, most would demonstrate a ratio at approximately 0.5 . It should be reiterated once again that the Ti-6-4 heattreatment conditions employed here were non-standard and stronger performance has been achieved in previous studies ${ }^{[7]}$.

Generally, the Ti-407 fractures appeared to display the more ductile appearance. When viewing all of the Ti-6-4 and Ti-407 fatigue fracture surfaces under high magnification SEM, irrespective of HCF/LCF/dwell loading waveforms, there was a clear correlation between the morphology of the pre-existing $a_{p}$ grains (Figure 1) and the individual quasi-cleavage facets clustered at the initiation sites, Figure 6. In Figure 6a, the shape of the Ti-6-4 facets is generally equi-axed, whereas in Figure $6 \mathrm{~b}$ the facets appear more elongated due to the form of the non-fully spheroidised Ti-407 $a_{p}$ grains. Under very close inspection, the central surface appearance of the individual facets was virtually featureless in both alloys. The area defined by the quasi-cleavage facet clusters was typically smaller in Ti-407 specimens compared to Ti-6-4, as illustrated by the two fractographs taken at identical magnification in Figure 6 (i.e. the facetted region in the Ti-6-4 shown in Figure 6a actually extended beyond the field of view).

Ancillary fatigue crack growth experiments have proven that the Stage II Paris fatigue crack growth characteristics for various microstructural forms of the Ti-407 alloy are similar to typical data for Ti-6-4 ${ }^{[8]}$. Therefore, the crack initiation phase appears to dominate the distinct differences in total fatigue life shown in Figure 2.
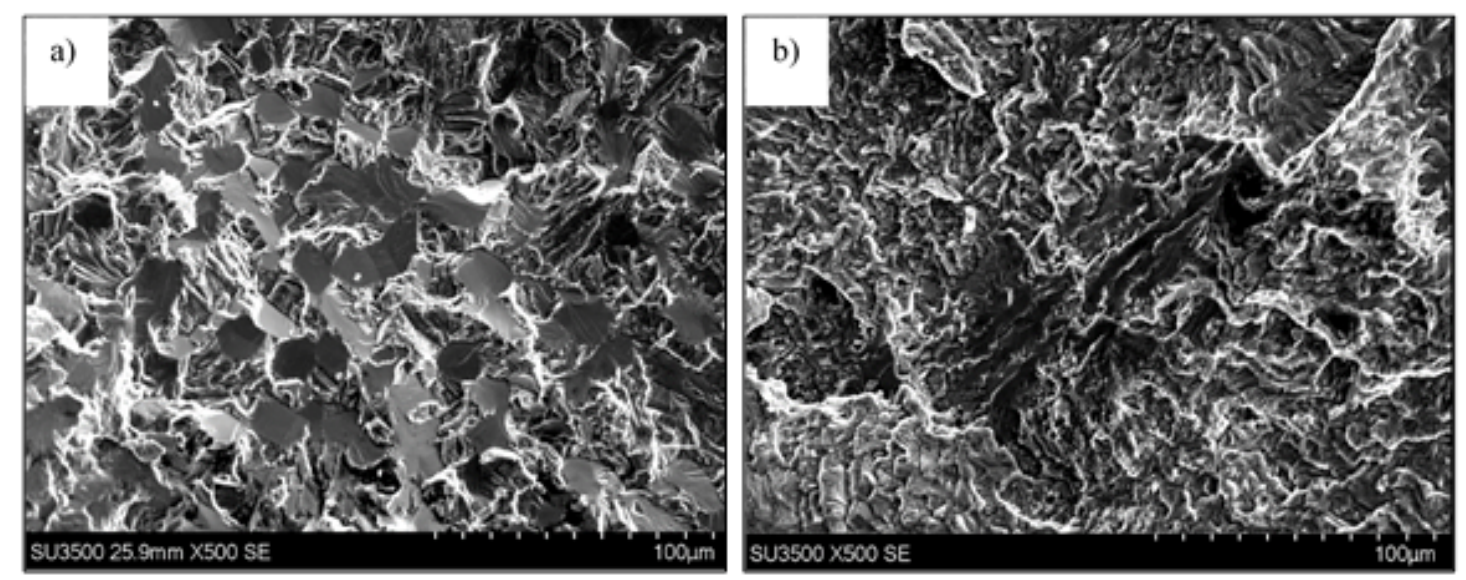

Electron back scattered diffraction (EBSD) mapping across a metallographic section extracted approximately $1 \mathrm{~mm}$ below the fracture surface of a single HCF specimen (Figure 7a) indicated extensive regions or "macrozones" containing a grains with a preferred basal crystallographic orientation. This suggests that adjacent macrozones may behave as the controlling structural units in textured materials rather than individual grains. This would remain consistent with the Stroh and Evans-Bache models describing stress redistribution ${ }^{[9,10]}$ even though these previous models were originally proposed with a single grain envisaged as the "hard region", where dislocation activity and slip is not easily accommodated. The adjacent "soft regions" containing prismatic or pyramidal orientated $a_{p}$ grains or transformed $b$, would be more favourable for plastic deformation. Dislocations could be accommodated across extensive slip planes between multiple, neighbouring grains, eventually developing a pile up at the interface between the soft and hard regions. Eventually, stress redistribution from the soft to the hard region increases the local stress level within the latter, initiating cracking in the hard macrozone. A revised interpretation of the Evans-Bache model based on macrozones is illustrated in Figure $7 \mathrm{~b}$. Clearly, this redistribution model is reliant on the time dependant motion of dislocations through the polycrystalline structure. In turn, this is self-consistent with the ranking of fatigue performance under HCF, LCF and dwell loading conditions, Figure 3.

Previous LCF and dwell fatigue studies performed at Swansea University for a range of $a+b$ and near a alloys have often demonstrated such fracture initiating facets are orientated near perpendicular to the applied tensile axis ${ }^{[11]}$. Detailed quantitative tilt fractography (QTF) following the techniques described by Sinha and Themelis ${ }^{[7,12]}$ was performed during the present study to measure the inclination of initiating facets relative to the loading direction. Once again, the facets were confirmed to be near perpendicular to the tensile stress axis under LCF or dwell loading, for either Ti-407 or Ti-6-4 specimens. However, facets developed in either alloy under HCF were typically angled between $30^{\circ}$ and $45^{\circ}$ to the applied load axis. This can be observed by the near surface initiation site illustrated in Figure 8, where the plane of early crack development, encompassing multiple basal facets, can be seen to be inclined to the loading direction. 

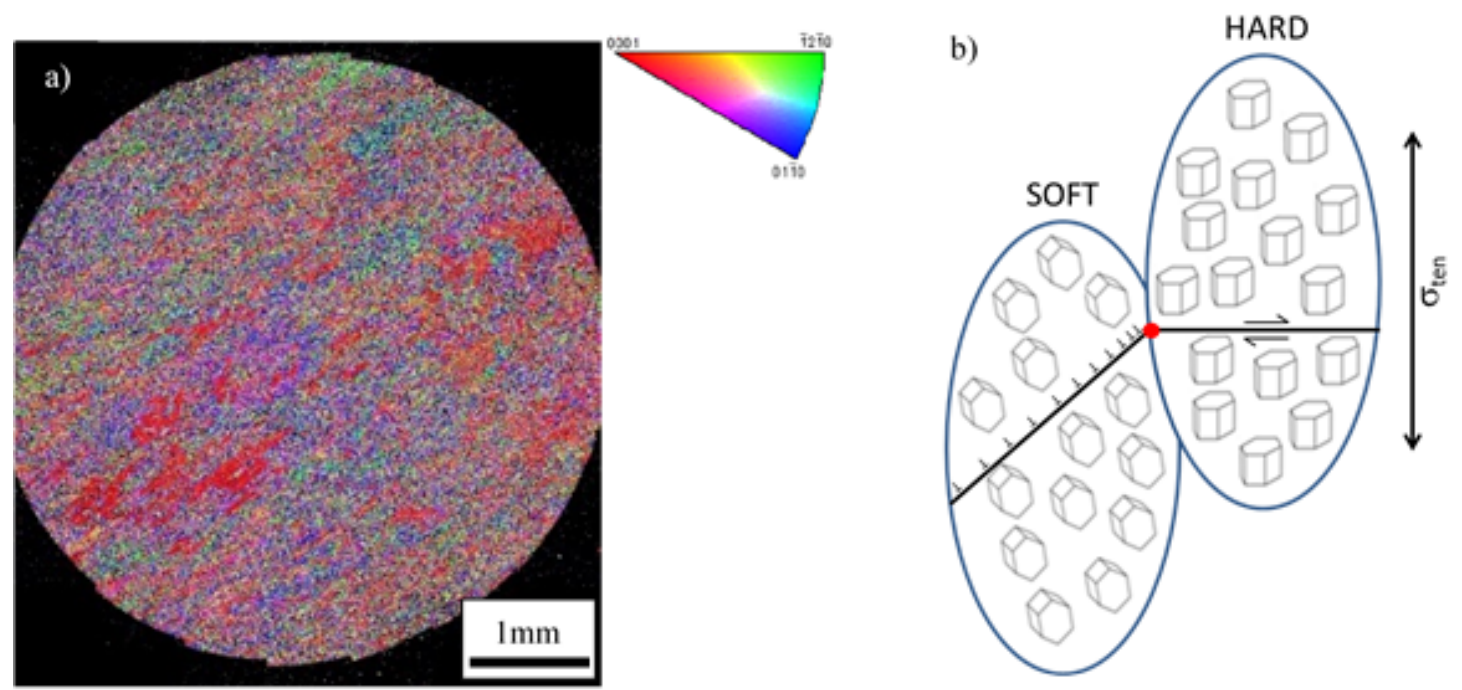

Fig.7. a) EBSD orientation map of a Ti-407 sub-fracture section; b) the Evans-Bache model applied to macrozones.
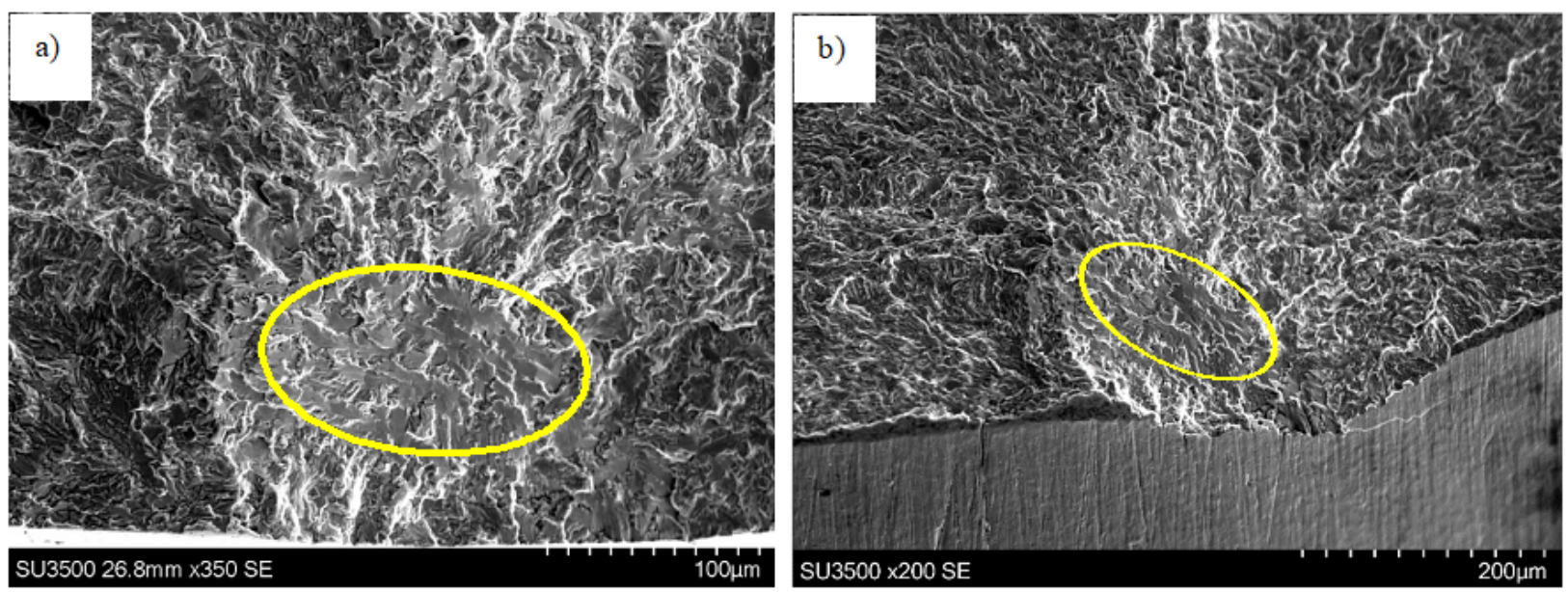

Figure 8. Near surface crack initiation and early stage growth under HCF. a) plan view of facets, b) side view.

Viewing the fatigue behaviour of Ti-407 in isolation (Figure 3), it was interesting to note the greater endurance strength measured under HCF conditions. Fatigue-creep interactions offer some insight into the difference between the HCF, LCF and dwell data. Whilst it would be difficult to imagine any creep influence from fatigue testing under a high frequency, under the LCF $15 \mathrm{cpm}$ trapezoidal loading and certainly under the dwell waveform "cold creep" damage can accumulate. In a similar fashion, Bache previously demonstrated that fatigue tests performed on the compressor disc alloy Ti-834 under sinusoidal waveforms of relatively higher frequencies induced a stronger response when compared to an LCF based $15 \mathrm{cpm}$ waveform ${ }^{[13]}$. That study went on to assess Ti-834 under a commonly employed two minute dwell waveform and showed forged variants of the alloy were particularly susceptible to "cold dwell". Creep strain accumulation was directly proportional to dwell periods. In light of the current investigation on Ti-407, the one second dwell at peak load during the $15 \mathrm{cpm}$ LCF experiments appears to induce attributes of a similar dwell response. The elongated form of the quasi-cleavage faceting in the Ti-407 fatigue specimens also draws comparisons to Ti- $834^{[14]}$. At the heart of the initiation sites, clusters of multiple facets were discernible, each associated with individual, elongated $\mathrm{a}_{\mathrm{p}}$ grains.

Referring back to Figure 3, the results of the Ti-407 dwell tests with a 120 s hold at maximum stress were presented. At an applied stress of $650 \mathrm{MPa}$, the 15 cycle per minute waveform delivered in excess of an order of magnitude longer life compared to the equivalent dwell test. However, it is noted that this magnitude of applied peak stress marginally exceeds the $0.2 \%$ proof stress of the alloy. The fractured dwell specimen tested at $650 \mathrm{MPa}$ demonstrated clear signs of bulk plasticity in the form of a reduced cross section and marked shear lips around the periphery, Figure 9. Assuming this reduction in area was generated from the first cycle onwards, the true applied stress acting on this specimen throughout the subsequent fatigue life was substantially higher than the engineering stress plotted in Figure 3. Therefore, this may be accentuating the apparent knockdown in dwell related performance at these elevated applied stress levels. The form of these fractures is also reminiscent of fatigue tests performed on Ti-6-4 at extremely high $\mathrm{R}$ ratio $(\mathrm{R}>0.8)^{[15]}$, indicating that the reduced strength and increased ductility of Ti-407 can promote similar plasticity under much reduced mean stress conditions. 
At lower levels of applied stress, the relative difference between Ti-407 performance under LCF and dwell fatigue lives is significantly reduced. A similar effect was demonstrated for Ti-834, with the point of merger between the separate data sets defined by the cyclic yield stress of the alloy ${ }^{[14]}$. A programme of strain controlled LCF testing has commenced to interrogate the same potential relationship in Ti-407.

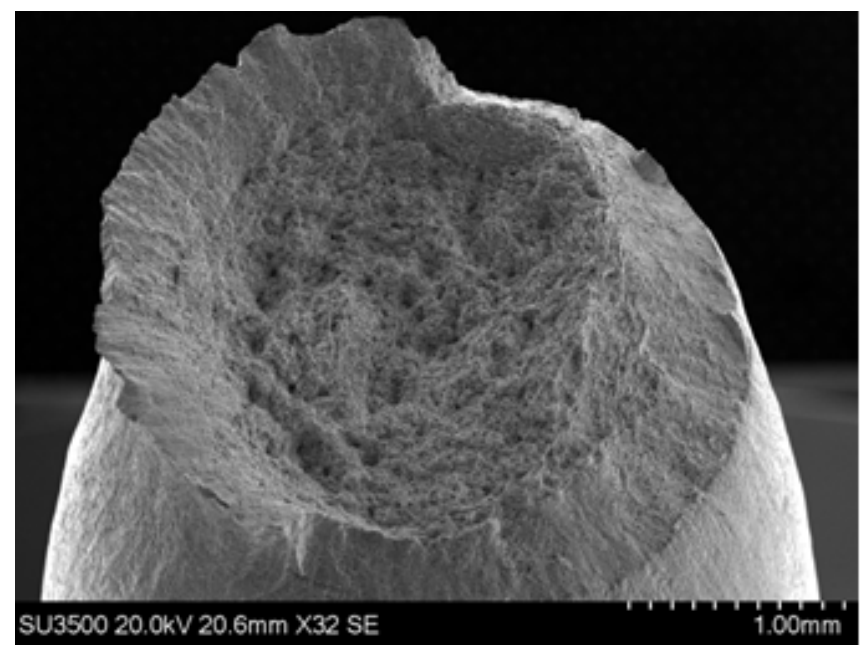

Figure 9: Ti-407 dwell fracture generated at $650 \mathrm{MPa}, \mathrm{R}=\mathbf{0}$.

The application of HCF loading on Ti-407 has demonstrated that a relatively strong fatigue response is measured under waveforms in the absence of any dwell period at the peak of the cycle. From a theoretical perspective, the HCF strength measured under a high frequency loading waveform could be considered to represent the ultimate fatigue performance, free of any influence from an additional contribution from creep damage. Alloys containing microstructures that are conducive to cold creep, extensive slip and stress redistribution mechanisms do not achieve this ultimate fatigue strength under LCF and dwell loading due to the "premature" initiation of facets orientated favourably for transfer to stage II crack growth under the action of a local Mode I stress. It has been argued that the $15 \mathrm{cpm}$ trapezoidal waveform employed during the current investigation to define LCF performance can itself be deemed as a "dwell" waveform, incorporating a 1 second hold at peak stress. Again, with reference to previous former studies conducted at Swansea University, it has been observed that this short hold period is sufficient to induce bulk stress relaxation events under individual strain controlled test cycles. Under the load controlled conditions employed here, the same mechanism would drive time dependent dislocation movement and generation of pile ups between hard/soft grains or macrozones, resulting in the "cold creep" induced dwell sensitive behaviour.

A notable result from this study is the exceptional HCF performance of Ti-407, particularly considering its relatively low yield strength compared to Ti-6-4. When HCF results for Ti-407 and the non-standard Ti-6-4 specimens are normalised relative to their measured $0.2 \%$ proof stress values, Figure 10 , it is seen that Ti-407 demonstrates a vastly superior response to both the Ti-6-4 variants tested.

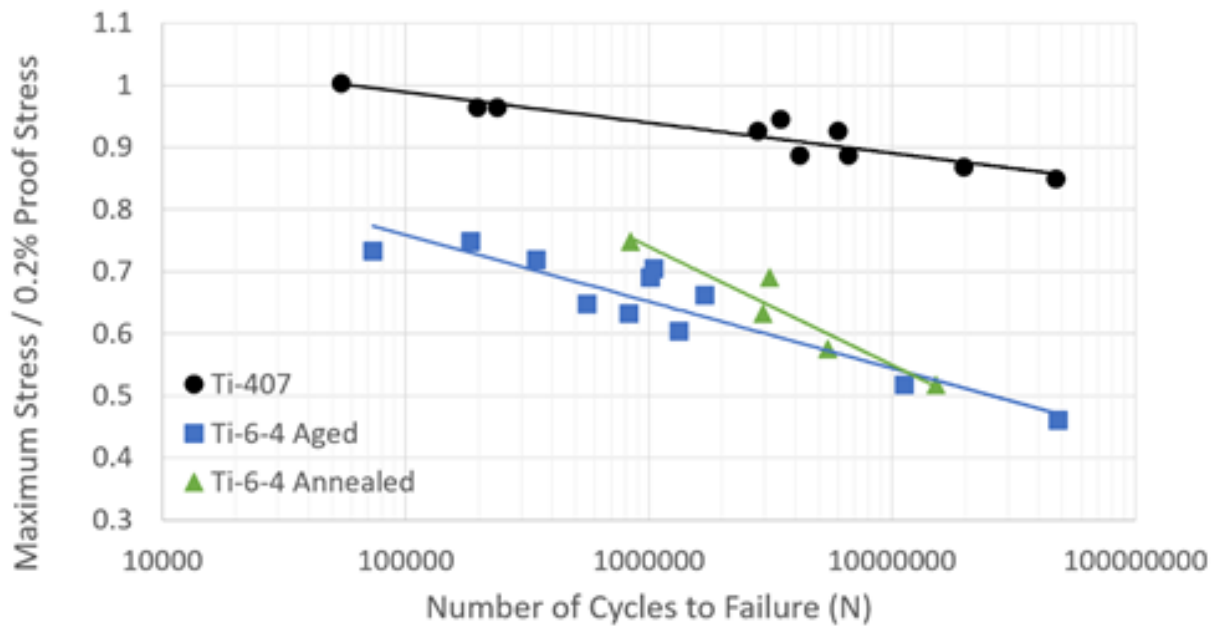

Figure 10. HCF performance as a function of $0.2 \%$ proof stress.

\section{Conclusion}

The following conclusions were drawn from the present research: 
- Compared to the non-standard variants of Ti-6-4 employed during the present study, Ti-407 offers a superior HCF fatigue strength.

- The fatigue performance of Ti-407 under HCF/LCF/dwell waveforms is associated with the relative superposition of "cold creep".

- EBSD data obtained from metallographic sections of Ti-407 indicated the presence of macrozones - regions containing grains with common basal plane orientation.

- Quasi-cleavage facets were invariably noted at crack initiation sites in Ti-407 specimens, including examples where failure initiated sub-surface.

- Clusters of facets appeared similar in morphology to the underlying microstructure, including elongated ap grains with transformed product between.

- Quantitative tilt fractography performed on facets exposed on the fatigue fracture surfaces, demonstrated that facets generated under $\mathrm{HCF}$ were inclined between $30^{\circ}$ and $45^{\circ}$ to the tensile load axis. This contrasted to the orthogonal facets noted under $15 \mathrm{cpm}$ LCF and dwell loading waveforms.

- The alloy TIMETAL $® 407$ should offer improved performance over Ti-6-4 in various engineering applications where the key design criteria is HCF endurance strength.

\section{References}

[1] R.A. Wood and R.J. Favor, Titanium Alloys Handbook. Ohio, 1972.

[2] H. Matsumoto et al, J. Alloy. Compunds, 509, (2011), pp. 2684-2692.

[3] S. James et al, in Proc. 13th World Conference on Titanium, 2016. DOI: 10.1002/9781119296126.ch121.

[4] G. Lütjering, Mater. Sci. Eng. A, vol. 243, no. 1-2, pp. 32-45, 1998.

[5] M. T. Whittaker et al, Int. J. Fatigue, vol. 31, no. 11-12, pp. 2022-2030, 2009.

[6] http://asm.matweb.com/search/SpecificMaterial.asp?bassnum=MTA321, [Accessed: 31-Oct-2018]

[7] V. Sinah, M. . Mills, and J. . Williams, J. Mater. Sci., vol. 42, no. 19, pp. 8334-8341, 2007.

[8] M.R. Bache, W.J. Evans and M. McElhone; Mat. Sci. and Eng., A234-236 (1997), pp.918-922.

[9] A. N. Stroh, Proc. R. Scociety A Mathmatical, Phys. Eng. Sci., vol. 223, no. 1154, pp. 404-414, 1954.

[10] W. J. Evans and M. R. Bache, in Seventh World Conference on Titanium TMS, 1993, pp. 1693-1700.

[11] M. R. Bache, Int. J. Fatigue, vol. 25, no. 9-11, pp. 1079-1087, 2003.

[12] G. Themelis, S. Chikwembani, and J. Weertman, Mater. Charact., vol. 24, no. 1, pp. 27-40, 1990.

[13] M.R.Bache, private data, Swansea University.

[14] M.R. Bache et al; Int. J. Fatigue, 1997;19(Suppl 1): pp.S83-S8.

[15] R.J. Morrissey, D.L. McDowell and T. Nicholas; Int. J. Fatigue, 21 (1999), pp. 679-685. 